\title{
Cutaneous Paraneoplastic Manifestation (MoRPHEA, LiCHEN SClERosus) - TWO CASE REPORTS
}

\author{
Pappova $\mathrm{T}^{1}$, PeC $\mathrm{J}^{1}$, Kozarova $\mathrm{A}^{1}$, Adamicova $\mathrm{K}^{2}$ \\ ${ }^{1}$ Comenius University Jessenius Faculty of Medicine and University Hospital, Department of Dermatovenerology, \\ Martin, Slovak Republic \\ ${ }^{2}$ Comenius University, Jessenius Faculty of Medicine and University Hospital, Department of Pathology, Martin, \\ Slovak Republic
}

\begin{abstract}
Internal malignancy may be presented in the form of paraneoplastic syndromes, which may indicate either formation or recurrence of a previously treated malignancy. Furthermore cutaneous paraneoplastic disorders often precede a diagnosis of cancer. We present 2 unique case reports with cutaneous paraneoplastic manifestations. The first one describes a patient with sudden progression of long-term stabilized morphea in connection with newly diagnosed hepatocellular carcinoma (HCC). The second one describes female patient with breast cancer preceded by the development of extragenital lichen sclerosus (LS) with typical sclerotic lesions and hemorrhagic bullae.
\end{abstract}

Key words: Paraneoplastic syndroms, bullous lichen sclerosus, morphea

\section{INTRODUCTION}

Paraneoplastic syndromes are rare disorders which arise as a result of the presence of cancer in the body and may affect diverse organ systems. They arise from tumor secretion of hormones, cytokines, biologically active proteins and their precursors or from immune cross-reactivity between malignant and normal tissues. Up to date the most commonly associated malignancies include small cell lung cancer, breast cancer, gynaecologic tumors, and hematologic malignancies. On average, it is estimated, that $8 \%$ of patients with cancer may present this type of tumor manifestation. As diagnostic methods have improved and patients live longer, the incidence of paraneoplastic syndromes is gradually increasing $(1,2)$. We report two clinical cases with cutaneous paraneoplastic manifestations. The first one discusses morphea and the second one LS with bullae formation as a paraneoplastic manifestation. These disorders either started or have got significantly worsen regarding to newly diagnosed cancer.

\section{Case report 1}

A 68-years-old male patient was admitted to our department because of sudden progression of previously stabilised morfea for more than 20 years. Upon physical examination, in addition to generalised old hyperpigmented lesions, there were numerous new sclerotic lesions with lilac ring (Fig.1). Over the last year, he underwent two times MRI because of hepar cirrhosis associated with small nodules suspected to be highly dysplastic with possible development of HCC in the segment VII. Moreover splenomegaly and portal hypertension with partial thrombosis of portal vein were repeatedly noticed. According to cirrhosis, biochemical parameters revealed hepatopathy by elevated GMT, total and conjugated bilirubin and complete blood count was with severe thrombocytopenia. Viruses of hepatitis A, B and C were investigated to exclude a viral etiology of cirrhosis, but all of them were negative.

\footnotetext{
Address for correspondence:

T. Pappova MD, Department of Dermatovenerology, Jessenius Faculty of Medicine and University Hospital, Kollarova Str. N.2, 03601 Martin, Slovak Republic

e-mail: tana.pappova@gmail.com
} 
Although AFP as a specific tumor marker for HCC was negative, histological examination of hepatic sample revealed a high grade dysplastic nodule and, marginally, there were signs of early state of HCC (Fig. 2). Histological examination of sclerodermic cutaneous lesion confirmed the diagnosis of morphea (Fig. 3). Systemic involvement was excluded by numerous examinations such as creatinine clearance to measure kidney function, echocardiogram and spirometry to assess lung function.

Fig. 1 Widespread lesions of morphea surrounded by lilac ring

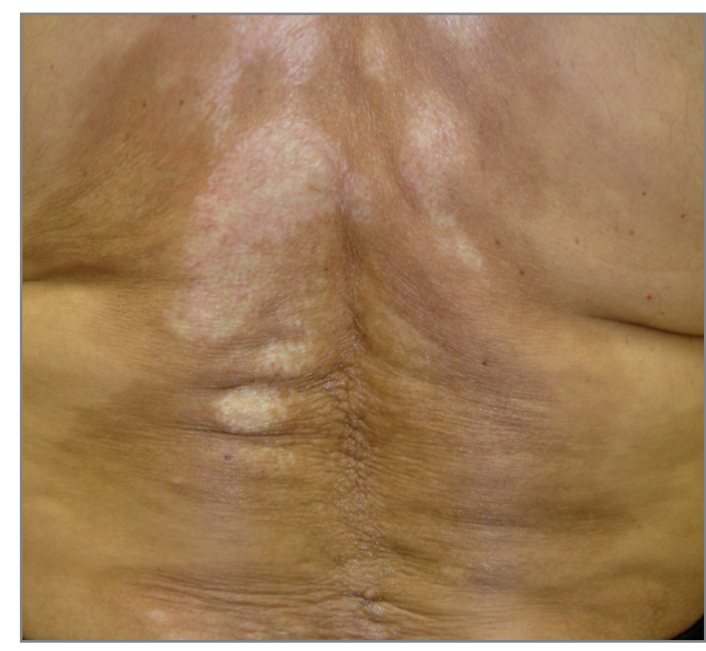

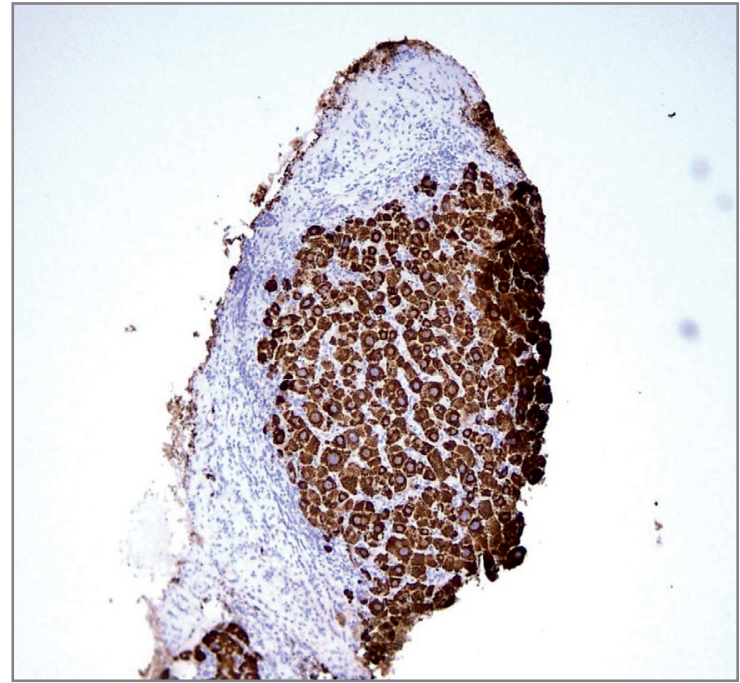

Fig. 2 Histology of hepatic tumor: high cytoplasmic positivity of abnormal hepatocytes (HepPar 1+, magnifying 150x)

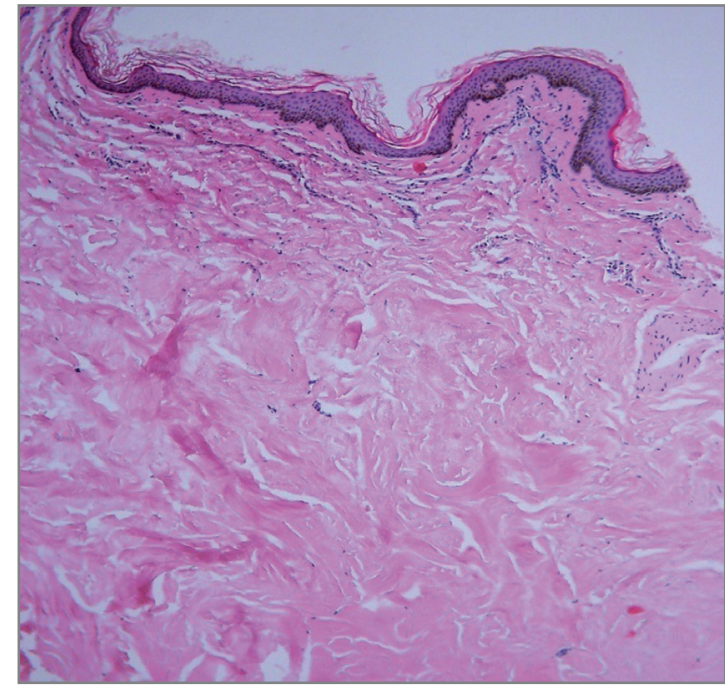

Fig. 3 Histology of the skin: Thickened collagen bundles (HE, magnifying 100x)

\section{Case report 2}

53-years-old female patient was treated for several hypopigmented and hyperpigmented macules localised on the torso, groins, shins and shoulders, which were first clinically classified as localized scleroderma. According to the progression of these lesions and positive serology for 
active infection caused by Borrelia burgdorfferi in association with localised scleroderma, she received antibiotic treatment with benzylpenicillin, for 21 days. Therapeutic effect of antibiotic treatment was only partial and temporary. One year later, a ductal invasive breast carcinoma was diagnosed, followed by ablation of her left breast with exenteration of lymph nodes and in 9 of 12 lymph nodes, the metastatic involvement was detected. Considering these findings, she received radiotherapy and chemotherapy. Other metastases were excluded by PET/CT examination. Over the course of this year, the skin changes were in progression with bullae formation and she was referred to our department because of failure of acyclovir therapy for previously suspected herpes zoster. Dermatologic examination revealed multiple hypopigmented atrophic macules, clinically re-diagnosed as an extragenital form of LS (Fig. 4) and extensive haemorrhagic bullae covered the whole area, where the radiotherapy had been applied (Fig. 5). Bullous form of LS was verified by histologic examination of bulla (Fig. 6). Because of oncology diagnosis, we established tumor markers as CEA and CA 19-9. CEA demonstrated increasing tendency and CA 19-9 was highly elevated compared to outpatient laboratory findings.

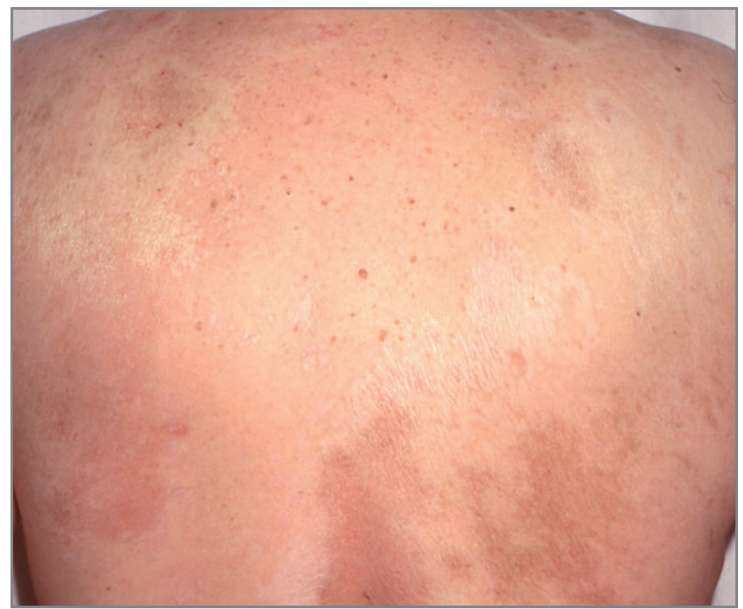

Fig.4 Hypopigmented atrophic and hyperpigmented lesions

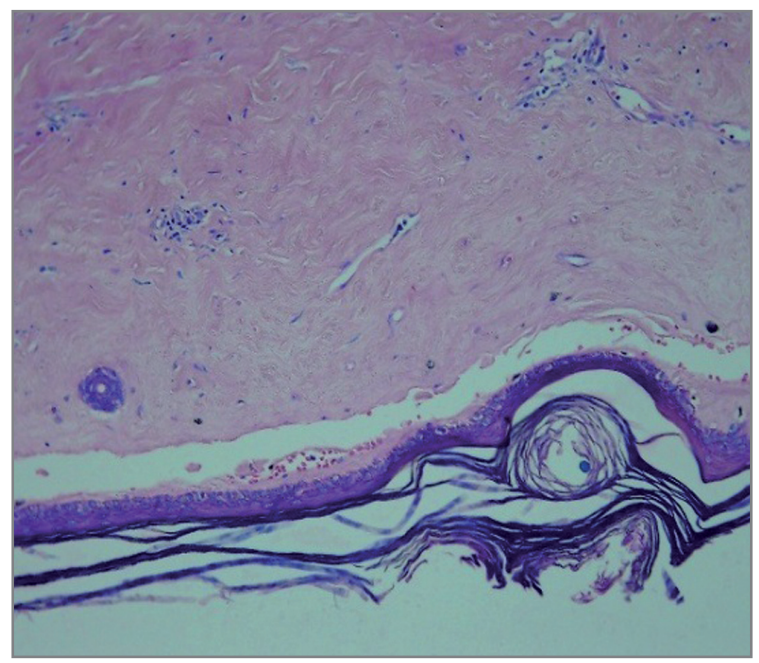

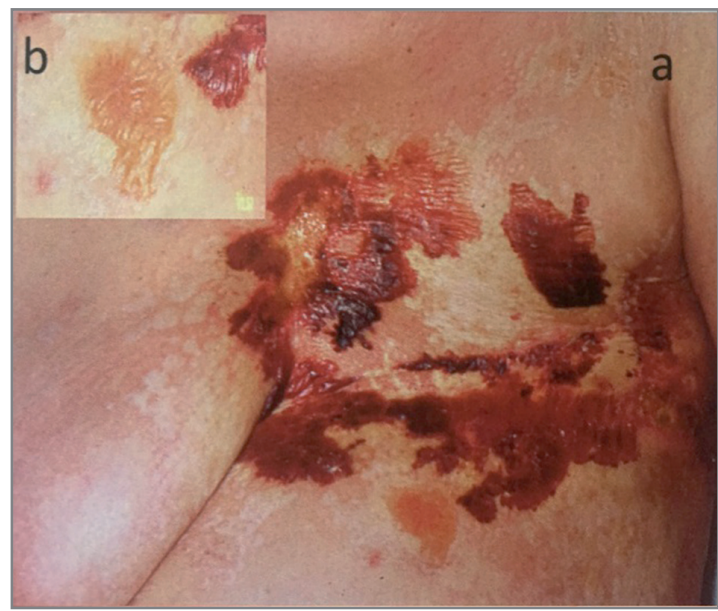

Fig. 5 a - haemorrhagic bullae at the site of radiation, $b$ - detail of the bulla

Fig.6 Histology of the bulla: suepidermal blister, epidermal atrophy and homogenisation of the collagen (HE, magnifying 100x) 


\section{DISCUSSION}

Malignancy associated with systemic scleroderma has been described as a direct consequence of fibrosing process involving internal organ as well as an origin of paraneoplastic manifestation predominately linked with lung cancer, liver cancer, urinary tract or malignant hematologic disorders $(3,4)$. Up to date, several studies have pointed at sudden development of morphea before or in brief time interval after the diagnosis of malignancy. These disorders were the most commonly observed in association with lung and breast cancer $(5,6,7,8)$ supporting the theory of paraneoplastic etiology of morphea.

In our study, examined patient suffered from a localised type of scleroderma without confirming the markers for systemic involvement. For mentioned reasons, it is less likely that morphea can lead to the development of HCC. Progression of sclerotic skin changes with the absence of systemic involvement in new diagnosed HCC suggests the probability of paraneoplastic symptom.

The etiopathogenesis of LS is not clearly understood, but there is a general consensus of autoimmune basis, which is also supported by the fact that the LS is frequently associated with other autoimmune diseases such as autoimmune thyreoiditis, alopecia areata, vitiligo or pernicious anemia (9). Localisation of LS may be influenced by Koebner phenomenon, which means that lesions form in different areas after trauma, on the site of chronic friction or in scars. Radiotherapy is also considered to provoke traumatisation and sclerotization of the skin. Few publications reported the development of $\operatorname{LS}$ in radiated area $(10,11,12,13)$. Here Curth supposed paraneoplastic form of LS and described patients with unspecified internal malignancies whose LS was ameliorated with tumor resection and flared with tumor recurrence (13).

However, in presented patient the diagnosis of bullous form of LS was made by histological verification from irradiated area, disseminated lesions which developed at the time of cancer diagnosis, clinically classified as LS, spared from radiotherapy, point at the paraneoplastic origin of extragenital LS. Moreover, sudden progression of cutaneous changes in form of bullae formation may signalize recurrence of breast cancer. This hypothesis is also supported by the presence of tumor markers with increasing tendency.

\section{CONCLUSION}

The etiopathogenesis of LS and morphea is still not fully understood, but more and more the authors emphasize the autoimmune processes. Since some paraneoplastic manifestations develop as a result of cross reaction of autoimmune mechanisms with production of antibodies, between malignant and healthy tissue, it is probable that mentioned case reports may prove paraneoplastic origin of these dermatological disorders.

\section{REFERENCES}

1. Pelosof L, Gerber DE. Paraneoplastic Syndromes: An Approach to Diagnosis and Treatment. Mayo Clin Proc. 2010; 85 (9): 838-854.

2. Šandorová M. Paraneoplastické syndrómy. Onkológia. 2010; 5 (6): 348 - 351.

3. Forbes AM, Woodrow JC et al. Carcinoma of brest and scleroderma: four further cases and a literature review. British journal of rheumatology. 1989; 28 (1): 65-69

4. Onishi A, Sugiyama D. et al. Cancer Incidence in Systemic Sclerosis: Meta-Analysis of Population-Based Cohort Studies. Arthritis \& Rheumatism. 2013; 65 (7): 1913-1921.

5. Benekli M, Yalcin B et al. Disseminated Morphea in Small Cell Lung Cancer. Acta Derm Venereol. 1998; 78 (1): 71-2.

6. Davis DA, Cohen PR et al. Localized scleroderma in breast cancer patients treated with supervoltage external beam radiation: Radiation port scleroderma. Journal of the American Academy of Dermatology. 1996; 35 (6): 923-927. 
7. Desmond BL, Blattner CM, Young J. Generalized morphea as the first sign of breast carcinoma: a case report. Dermatology Online Journal. 2016; 22 (2): 10.

8. Omid J, Roxann P et al. Paraneoplastic generalized progressive bullous morphea. Journal of the American Academy of Dermatology. 2015; 72 (5)S1, p. AB57.

9. Fistarol S. K., Itin P.H.: Diagnosis and Treatment of Lichen Sclerosus. Am J Clin Dermatol. 2013; 14 (1): 27-47.

10. Tournillac I, Dandurand M, Guillot B. Bullous lichen sclerosus after radiotherapy. Ann Dermatol Venereol. 1998; 125 (2): 121-3.

11. Vujovic O, Lichen sclerosus in a radiated breast. CMAJ. 2010; 182 (18): p. E860.

12. Yates VM, King CM, Dave VK. Lichen sclerosus et atrophicus following radiatioon therapy. Arch Dermatol. 1985; 121: 1044-7.

13. Curth HO. Lichen sclerosus et atrophicus [Comment]. Arch Dermatol. 1973; 108: 433.

Received: March, 3, 2017

Accepted: March, 21, 2017 